\title{
CONTRIBUTIONS FROM CONJUGACY CLASSES OF REGULAR ELLIPTIC ELEMENTS IN HERMITIAN MODULAR GROUPS TO THE DIMENSION FORMULA OF HERMITIAN MODULAR CUSP FORMS ${ }^{1}$
}

BY

MINKING EIE

\begin{abstract}
The dimension of the vector space of hermitian modular cusp forms on the hermitian upper half plane can be obtained from the Selberg trace formula; in this paper we shall compute the contributions from conjugacy classes of regular elliptic elements in hermitian modular groups by constructing an orthonomal basis in a certain Hilbert space of holomorphic functions. A generalization of the main Theorem can be applied to the dimension formula of cusp forms of $S U(p, q)$. A similar theorem was given for the case of regular elliptic elements of $\operatorname{Sp}(n, \mathbf{Z})$ in [5] via a different method.
\end{abstract}

1. Introduction and notation. Denote by $E$ the unit matrix and by 0 the zero matrix in the matrix ring $M_{n}(\mathbf{C})$. Put $J=\left[\begin{array}{cc}0 & E \\ -E & 0\end{array}\right]$. The hermitian symplectic group of degree $n, \Omega_{n}$, is then defined as the group of matrices in $M_{2 n}(\mathbf{C})$; it satisfies ${ }^{t} \bar{M} J M=J$; i.e.

$$
\Omega_{n}=\left\{\left.M \in M_{2 n}(\mathbf{C})\right|^{t} \bar{M} J M=J\right\} .
$$

Here ${ }^{t} \bar{M}$ is the transpose complex conjugate to $M$.

Let $\mathcal{H}_{n}$ be the hermitian upper half plane; specifically,

$$
\mathscr{H}_{n}=\left\{Z \in M_{n}(\mathbf{C}) \mid Z=X+i Y, X={ }^{t} \bar{X}, Y={ }^{t} \bar{Y}>0\right\} \text {. }
$$

The hermitian symplectic group $\Omega_{n}$ operates on $\nvdash_{n}$ transitively by the action

$$
M: Z \rightarrow M(Z)=(A Z+B)(C Z+D)^{-1}, \quad M=\left[\begin{array}{cc}
A & B \\
C & D
\end{array}\right] \in \Omega_{n} .
$$

For a given imaginary quadratic number field $\mathbf{F}$, we denote by $\mathbf{K}$ its ring of integers. The hermitian modular group of degree $n, \Gamma_{n}(\mathbf{K})$, is defined as

$$
\Gamma_{n}(\mathbf{K})=\Omega_{n} \cap M_{2 n}(\mathbf{K}) .
$$

An element $M$ in $\Gamma_{n}(\mathbf{K})$ is regular elliptic if $M$ has an isolated fixed point on $\nvdash_{n}$, i.e. the equation $M(Z)=Z$ has a unique solution on $\mathscr{H}_{n}$. A similar argument as in [5] shows that the following statements are equivalent:

(1) $M$ is a regular elliptic element in $\Gamma_{n}(\mathbf{K})$ and its characteristic polynomial $\varphi(X)$ is in $Z[X]$.

(2) $M \in \Gamma_{n}(\mathbf{K})$ and is conjugate in $\Omega_{n}$ to $\operatorname{diag}\left[\lambda_{1}, \lambda_{2}, \ldots, \lambda_{n}, \bar{\lambda}_{1}, \bar{\lambda}_{2}, \ldots, \bar{\lambda}_{n}\right] ; \lambda_{i}$ $(i=1,2, \ldots, n)$ are roots of unity and $\lambda_{i} \lambda_{j} \neq 1$ for all $i, j$.

Received by the editors January 7, 1985 .

1980 Mathematics Subject Classification. Primary 10D20, 10 D05.

${ }^{1}$ Research supported by Academia Sinica and NSC of Taiwan, Republic of China. 
Let $S\left(k ; \Gamma_{n}(\mathbf{K})\right)$ denote the space of holomorphic functions $f(Z)$ on $\mathscr{H}_{n} ; f(Z)$ satisfies the following conditions:

(1) $f(M(Z))=\operatorname{det}(C Z+D)^{k} f(Z)$ for all $M=\left[\begin{array}{ll}A & B \\ C & D\end{array}\right]$ in $\Gamma_{n}(\mathbf{K}), Z \in \not_{n}$.

(2) $(\operatorname{det} Y)^{k / 2} f(Z)$ is bounded on $\nvdash_{n}, Z=X+i Y$.

A function in $S\left(k ; \Gamma_{n}(\mathbf{K})\right)$ is called a hermitian modular cusp form of weight $k$ and degree $n$.

For fixed degree $n$ and certain $k$, the first condition may be satisfied only for $f(Z)=0$. However we shall exclude these trivial cases. For example, we assume $k n \equiv 0(\bmod 4)$ when $\mathbf{K}=\mathbf{Z}[i]$ and $\mathbf{F}=\mathbf{Q}[i]$. It is well known that $S\left(k, \Gamma_{n}(\mathbf{K})\right)$ is a finite dimensional Hilbert space [6]. Furthermore, its dimension can be written as an integral of a Bergman kernel function on a certain Hilbert space over the fundamental domain in $\not_{n}$ with respect to $\Gamma_{n}(\mathbf{K})$, when $k$ is sufficiently large (for example $k>(4 n-2)$; see also [9]). This is the so-called Selberg trace formula.

More precisely, let $K\left(Z_{1}, Z_{2}\right)$ be a kernel function of the space $H\left(k ; \mathscr{H}_{n}\right)$ which consists of a holomorphic function on $\mathcal{H}_{n}$ and satisfies

$$
\int_{\mathcal{H}_{n}}(\operatorname{det} Y)^{k-2 n}|f(Z)|^{2} d Z<\infty
$$

Then

$$
\operatorname{dim}_{\mathbf{C}} S\left(k ; \Gamma_{n}(\mathbf{K})\right)=\int_{\mathcal{F}_{n}} \sum_{\gamma \in \overline{\Gamma_{n}(\mathbf{K})}} K(Z, \gamma(Z)) \overline{j(\gamma, Z)}^{-k}(\operatorname{det} Y)^{k-2 n} d Z
$$

where

(1) $\overline{\Gamma_{n}(\mathbf{K})}$ is the quotient group $\Gamma_{n}(\mathbf{K}) / U$ with $U$ the center of $\Gamma_{n}(\mathbf{K})$,

(2) $\mathcal{F}_{n}$ is a fundamental domain in $\mathcal{H}_{n}$ with respect to $\Gamma_{n}(\mathbf{K})$,

(3) $j(\gamma, Z)=\operatorname{det}(C Z+D)$ if $\gamma=\left[\begin{array}{ll}A & B \\ C & D\end{array}\right] \in \Gamma_{n}(\mathbf{K})$,

(4) $Z=X+i Y$ in $\nvdash_{n}$ and $d Z=d X d Y$ is the Euclidean measure on $C^{n^{2}}$.

In this paper, we shall consider the subseries with the summation ranging over all regular elliptic elements in $\Gamma_{n}(\mathbf{K})$; or consider the contributions from conjugacy classes of regular elliptic elements to $\operatorname{dim}_{\mathbf{C}} S\left(k ; \Gamma_{n}(\mathbf{K})\right)$. We shall obtain the following

THEOREM. Suppose $M \in \Gamma_{n}(\mathbf{K})$ and is conjugate in $\Omega_{n}$ to

$$
\operatorname{diag}\left[\lambda_{1}, \lambda_{2}, \ldots, \lambda_{n}, \bar{\lambda}_{1}, \bar{\lambda}_{2}, \ldots, \bar{\lambda}_{n}\right]
$$

with $\lambda_{j}(j=1,2, \ldots, n)$ roots of unity and $\lambda_{1} \lambda_{j} \neq 1$ for all $1 \leq i, j \leq n$. Then the contribution to $\operatorname{dim}_{\mathbf{C}} S\left(k ; \Gamma_{n}(\mathbf{K})\right)(k>(4 n-2))$ of regular elliptic elements in $\Gamma_{2}(\mathbf{K})$ which are conjugate in $\Gamma_{n}(\mathbf{K}) / U$ to $M$ is given by

$$
N_{\{M\}}=\left|C_{M, \mathbf{Z}}\right|^{-1} \prod_{j=1}^{n} \bar{\lambda}_{j}^{k} \cdot \prod_{j, k=1}^{n}\left(1-\bar{\lambda}_{j} \bar{\lambda}_{k}\right)^{-1} .
$$

Here $C_{M, \mathbf{Z}}$ is the centralizer of $M$ in $\Gamma_{n}(\mathbf{K}) / U$ and $\left|C_{M, \mathbf{Z}}\right|$ is its order.

REMARK. Here we shall exclude those integers $k$ such that

$$
f(M(Z))=\operatorname{det}(C Z+D)^{k} f(Z), \quad M=\left[\begin{array}{cc}
A & B \\
C & D
\end{array}\right] \in \Gamma_{n}(\mathbf{K}),
$$

is satisfied only for $f(Z)=0$. 
2. The Selberg trace formula. Since $H_{n}$ is mapped biholomorphically onto the bounded domain

$$
D_{n}: W \in M_{n}(\mathbf{C}), \quad E-W^{t} \bar{W}>0,
$$

under the Cayley transform $W=(Z-i E)(Z+i E)^{-1}$, it suffices to consider $H\left(k ; D_{n}\right)$ instead of $H\left(k ; \nvdash_{n}\right) . H\left(k ; D_{n}\right)$ consists of holomorphic function $f(W)$ satisfying

$$
\int_{D_{n}} \operatorname{det}\left(E-W^{t} \bar{W}\right)^{k-2 n}|f(W)|^{2} d W<\infty
$$

The Bergmann kernel function for $H\left(k ; D_{n}\right)$ is given by the following propositions.

PROPOSITION 1 [11, THEOREM 3.3]. Let $\varphi_{1}, \varphi_{2}, \ldots, \varphi_{n}, \ldots$ be any orthonormal basis of the Hilbert space $H\left(k ; D_{n}\right)$. Then the series

$$
\sum_{n=1}^{\infty} \varphi_{n}(W) \overline{\varphi_{n}\left(W_{1}\right)}
$$

converges uniformly on each compact subset of $D_{n} \times D_{n}$. The sum, denoted by $K\left(W, W_{1}\right)$, is independent of the choice of orthonomal basis and

$$
f(W)=\int_{D_{n}} \operatorname{det}\left(E-W_{1}^{t} \bar{W}_{1}\right)^{k-2 n} K\left(W, W_{1}\right) f\left(W_{1}\right) d W_{1}
$$

for each $f \in H\left(k ; D_{n}\right)$.

Proposition $2[\mathbf{9}$, Lemma 2.1]. Suppose that $k>(4 n-2)$. Then the function $K\left(W, W_{1}\right)$ is given by

$$
K\left(W, W_{1}\right)=C(k, n) \operatorname{det}\left(E-W^{t} \bar{W}_{1}\right)^{-k}
$$

with

$$
C(k, n)=\pi^{-n^{2}} \prod_{0 \leq i, j \leq n-1}(k-2 n+1+i+j) .
$$

ProOF. The kernel function is a constant multiple of $\operatorname{det}\left(E-W^{t} \bar{W}_{1}\right)^{-k}$ by arguments similar to I of $[\mathbf{7}]$. The constant $C(k, n)$ is determined by

$$
C(k, n)^{-1}=\int_{D_{n}} \operatorname{det}\left(E-W^{t} \bar{W}\right)^{k-2 n} d W .
$$

3. Convergence of the series. Let $\Lambda=\operatorname{diag}\left[\lambda_{1}, \lambda_{2}, \ldots, \lambda_{n}\right]$ be a unitary matrix. As an element of a hermitian symplectic group, the operation of $\Lambda$ on $D_{n}$ is given by

$$
\Lambda: W \rightarrow \Lambda W \Lambda, \quad W \in D_{n},
$$

and

$$
K(W, \Lambda W \Lambda)_{\overline{j(\Lambda, W)}^{-k}}=C(k, n)(\operatorname{det} \Lambda)^{-k} \operatorname{det}\left(E-\bar{\Lambda} W \bar{\Lambda}^{t} \bar{W}\right)^{-k}
$$

Now we shall prove this function is absolutely integrable on $D_{n}$ with respect to the measure $\operatorname{det}\left(E-W^{t} \bar{W}\right)^{k-2 n} d W$ when $k>(2 n-1)$. 
LEMMA 1 [8, THEOREM 1, P. 266]. If $E-Z^{t} \bar{Z} \geq 0$ and $E-W^{t} \bar{W} \geq 0$, then

$$
\operatorname{det}\left(E-Z^{t} \bar{Z}\right) \operatorname{det}\left(E-W^{t} \bar{W}\right)+|\operatorname{det}(Z-W)|^{2} \leq\left|\operatorname{det}\left(E-Z^{t} \bar{W}\right)\right|^{2} .
$$

Equality holds only when $Z=W$.

LEMMA 2. If $\Lambda=\operatorname{diag}\left[\lambda_{1}, \lambda_{2}, \ldots, \lambda_{n}\right]$ with $\lambda_{i}(i=1,2, \ldots, n)$ roots of unity and $1-\lambda_{i} \lambda_{j} \neq 0$ for all $i, j$, then

$$
\operatorname{det}\left(E-\bar{\Lambda} W \bar{\Lambda}^{t} \bar{W}\right) \neq 0
$$

for all $W \in \bar{D}_{n}$.

ProOF. Applying the previous lemma with $Z=\bar{\Lambda} W \bar{\Lambda}=\left[\bar{\lambda}_{i} \bar{\lambda}_{j} w_{i j}\right]$, we get

$$
\left[\operatorname{det}\left(E-W^{t} \bar{W}\right)\right]^{2}+|\operatorname{det}(\bar{\Lambda} W \bar{\Lambda}-W)|^{2} \leq\left[\operatorname{det}\left(E-\bar{\Lambda} W \bar{\Lambda}^{t} \bar{W}\right)\right]^{2} .
$$

Now suppose $\operatorname{det}\left(E-\bar{\Lambda} W \bar{\Lambda}^{t} \bar{W}\right)=0$. Then it forces

$$
\operatorname{det}\left(E-W^{t} \bar{W}\right)=0 \text { and } \bar{\Lambda} W \bar{\Lambda}=W .
$$

From $\bar{\Lambda} W \bar{\Lambda}=W$ and our assumption on $\Lambda$, we get $W=0$, which contradicts $\operatorname{det}\left(E-W^{t} \bar{W}\right)=0$. This proves our assertion.

Proposition 3. Let $M \in \Gamma_{n}(\mathbf{K})$ and be conjugate in $\Omega_{n}$ to

$$
\operatorname{diag}\left[\lambda_{1}, \lambda_{2}, \ldots, \lambda_{n}, \bar{\lambda}_{1}, \bar{\lambda}_{2}, \ldots, \bar{\lambda}_{n}\right]
$$

$\lambda_{i}(i=1,2, \ldots, n)$ roots of unity and $\lambda_{i} \lambda_{j} \neq 1$ for all $i, j$. Then we have

(1) $\int_{D_{n}} \operatorname{det}\left(E-W^{t} \bar{W}\right)^{k-2 n}\left|\operatorname{det}\left(E-\bar{\Lambda} W \bar{\Lambda}^{t} \bar{W}\right)\right|^{-k} d W<\infty$ for $k>(2 n-1)$,

(2) the contribution $N_{\{M\}}$ in the Theorem is given by

$$
\begin{aligned}
N_{\{M\}}=C & (k, n)(\operatorname{det} \Lambda)^{-k}\left|C_{M, Z}\right|^{-1} \\
& \times \int_{D_{n}} \operatorname{det}\left(E-W^{t} \bar{W}\right)^{k-2 n} \operatorname{det}\left(E-\bar{\Lambda} W \bar{\Lambda}^{t} \bar{W}\right)^{-k} d W .
\end{aligned}
$$

Proof. (1) follows since $\operatorname{det}\left(E-W^{t} \bar{W}\right)^{k-2 n} d W$ is a bounded measure on $D_{n}$ if $k>(2 n-1)$ and $\operatorname{det}\left(E-\bar{\Lambda} W \bar{\Lambda}^{t} \bar{W}\right) \neq 0$ for all $W$ in $D_{n}$.

To prove (2), we let $\{M\}$ denote the conjugacy class in $\Gamma_{n}(\mathbf{K}) / U$ and which can be represented by $M$. Then we have

$$
N_{\{M\}}=\int_{\mathcal{F}_{n}}(\operatorname{det} Y)^{k-2 n} \sum_{\gamma \in\{M\}} K(Z, \gamma(Z)) \overline{j(\gamma, Z)}^{-k} d Z .
$$

Note that the integral

$$
N=\int_{\mathcal{H}_{n}}(\operatorname{det} Y)^{k-2 n} K(Z, M(Z)) \overline{j(M, Z)}^{-k} d Z
$$

is transformed into

$$
(\operatorname{det} \Lambda)^{-k} C(k, n) \int_{D_{n}} \operatorname{det}\left(E-W^{t} \bar{W}\right)^{k-2 n} \operatorname{det}\left(E-\bar{\Lambda} W \bar{\Lambda}^{t} \bar{W}\right)^{-k} d W
$$


under the Cayley transform $W=(Z-i E)(Z+i E)^{-1}$. Now with (1), we know the integral $N$ is absolutely convergent. Hence we have

$$
\begin{aligned}
N & =\int_{\mathcal{F}_{n}}(\operatorname{det} Y)^{k-2 n} \sum_{\gamma \in \overline{\Gamma_{n}(K)}} K\left(Z, \gamma^{-1} M \gamma(Z)\right) \overline{j\left(\gamma^{-1} M \gamma, Z\right)}-k d Z \\
& =\left|C_{M, \mathbf{Z}}\right| \int_{\mathcal{F}_{n}} \sum_{\gamma \in\{M\}} K(Z, \gamma(Z)) \overline{j(\gamma, Z)}-k d Z=\left|C_{M, \mathbf{z}}\right| \cdot N_{\{M\}} .
\end{aligned}
$$

This proves our assertion in (2).

REMARK. Here we use the fact that the centralizer $C_{M, \mathbf{Z}}$ of $M$ in $\overline{\Gamma_{n}(\mathbf{K})}$ is a group of finite order since it is discrete and is conjugate in $\Omega_{n}$ to a subgroup of a unitary group which is compact.

4. Proof of the Theorem. To prove our Theorem, by Proposition 3 it suffices to evaluate the integral

$$
C(k, n) \int_{D_{n}} \operatorname{det}\left(E-W^{t} \bar{W}\right)^{k-2 n} \operatorname{det}\left(E-\bar{\Lambda} W \bar{\Lambda}^{t} \bar{W}\right)^{-k} d W .
$$

But this is not easy when $n \geq 2$. Here we shall first construct a new orthonomal basis in $H\left(k ; D_{n}\right)$.

LEMMA 3 [15, LEMMA 1, P. 27]. Let $S=\left[s_{i j}\right]$ be an $n \times n$ hermitian matrix, i.e. $s_{i j}=\bar{s}_{j i}$ for all $i, j$, and let $S_{j}(j=1,2, \ldots, n-1)$ be the submatrix consisting of $j \times j$ entries on the upper left block of $S$. Then $S$ is positively definite if and only if

$$
\operatorname{det} S>0 \quad \text { and } \quad \operatorname{det} S_{j}>0 \quad(j=1,2, \ldots, n-1) .
$$

Proposition 4. Let $\theta_{1 j}, \theta_{j 1}(j=1,2,3, \ldots, n)$ be $2 n-1$ real numbers and $W^{\prime}=\left[w_{j k}^{\prime}\right] \in D_{n}$. Suppose $W=\left[w_{j k}\right] \in M_{n}(\mathbf{C})$ is defined by

$$
\begin{cases}w_{j k}=w_{j k}^{\prime} e^{i \theta_{j k}}, & j=1 \text { or } k=1, i=\sqrt{-1}, \\ w_{j k}=w_{j k}^{\prime} e^{i\left(\theta_{j 1}+\theta_{1 k}-\theta_{11}\right)}, & j \neq 1 \text { and } k \neq 1 .\end{cases}
$$

Then we have $W \in D_{n}$. shows

ProOF. Let $E-W^{t} \bar{W}=\left[a_{j k}\right]$ and $E-W^{\prime t} \bar{W}^{\prime}=\left[b_{j k}\right]$. A direct calculation

(1) $a_{j k}=\overline{a_{k j}}, b_{j k}=\overline{b_{k j}}$ for all $k, j$;

(2) $a_{j j}=b_{j j}>0, j=1,2, \ldots, j$;

(3) $a_{j k}=b_{j k} e^{i\left(\theta_{j 1}-\theta_{k 1}\right)}$ for all $j, k$.

If $W^{\prime} \in D_{n}$, then the submatrix $W_{n-1}^{\prime}$ obtained from cancellation of the $n$th row and $n$th column of $W$ is in $D_{n-1}$. Thus by Lemma 3 and an induction on $n$, it suffices to prove $\operatorname{det}\left(E-W^{t} \bar{W}\right)>0$. But it is easy to show

$$
\operatorname{det}\left(E-W^{t} \bar{W}\right)=\operatorname{det}\left(E-W^{\prime} \bar{W}^{\prime}\right)
$$

by properites (1)-(3) and elementary properties of the determinant. This proves our assertion. 
For each $n^{2}$-tuple of nonnegative integers $\alpha=\left[\alpha_{j k}\right], 1 \leq j, k \leq n$; we shall let $W^{\alpha}$ denote the monomial

$$
\prod_{j, k=1}^{n} w_{j k}^{\alpha_{j k}}
$$

in the variable $W$, and let $|\alpha|=\sum_{j, k=1}^{n} \alpha_{j k}$ be the degree of $W^{\alpha}$.

Proposition 5. Let $W^{\alpha}$ and $W^{\beta}$ be monomials in $w_{j k}(j, k=1, \ldots, n)$. Then

$$
\int_{D_{n}} \operatorname{det}\left(E-W^{t} \bar{W}\right)^{k-2 n} W^{\alpha} \bar{W}^{\beta} d W=0
$$

unless

$$
\left\{\begin{array}{l}
\alpha_{11}-\beta_{11}+\sum_{j, k \geq 2}\left(-\alpha_{j k}+\beta_{j k}\right)=0, \\
\sum_{j=1}^{n}\left(\alpha_{j k}-\beta_{j k}\right)=0, \sum_{j=1}^{n}\left(\alpha_{k j}-\beta_{k j}\right)=0, \quad(k=2,3, \ldots, n) .
\end{array}\right.
$$

Under the above conditions, we have $|\alpha|=|\beta|$ and

$$
\sum_{j=1}^{n}\left(\alpha_{j k}+\alpha_{k j}\right)=\sum_{j=1}^{n}\left(\beta_{j k}+\beta_{k j}\right), \quad(k=1,2, \ldots, n) .
$$

PROOF. By the previous proposition, we can use polar coordinates on certain entries of $W$ as follows:

$$
\begin{cases}w_{j k}=r_{j k} e^{i \theta_{j k}}, & j=1 \text { or } k=1, r_{j k} \geq 0,0 \leq \theta_{j k}<2 \pi \\ w_{j k}=w_{j k}^{\prime} e^{i\left(\theta_{j 1}+\theta_{1 k}-\theta_{11}\right)}, & j \neq 1 \text { and } k \neq 1\end{cases}
$$

Let $D_{n}^{\prime}$ be the subset of $D_{n}$ and be defined by

$$
D_{n}^{\prime}: W=\left[w_{j k}\right] \in D_{n}, \quad w_{j 1}, w_{1 j} \geq 0(j=1,2, \ldots, n) .
$$

With these new coordinates, we have

$$
\begin{aligned}
\int_{D_{n}} \operatorname{det}\left(E-W^{t} \bar{W}\right)^{k-2 n} W^{\alpha} \bar{W}^{\beta} d W \\
=\int_{D_{n}^{\prime}} \operatorname{det}\left(E-W^{\prime} \bar{W}^{\prime}\right)^{k-2 n} \prod_{j=1}^{n} r_{1 j}^{\alpha_{1 j}+\beta_{1 j}+1} d r_{1 j} \\
\quad \times \prod_{j=2}^{n} r_{j 1}^{\alpha_{j 1}+\beta_{j 1}+1} d r_{j 1} \prod_{j, k \geq 2} w_{j k}^{\alpha_{j k} \bar{w}_{j k}^{\beta_{j k}} d w_{j k}} \\
\quad \times \int_{0}^{2 \pi} \exp \left[i \theta_{11}\left(\alpha_{11}-\beta_{11}+\sum_{j, k \geq 2}\left(-\alpha_{j k}+\beta_{j k}\right)\right)\right] d \theta_{11} \\
\quad \times \prod_{k=2}^{n} \int_{0}^{2 \pi} \int_{0}^{2 \pi} \exp \left[i \theta_{1 k}\left(\sum_{j=1}^{n}\left(\alpha_{j k}-\beta_{j k}\right)\right)\right] \\
\times \exp \left[i \theta_{k 1}\left(\sum_{j=1}^{n}\left(\alpha_{k j}-\beta_{k j}\right)\right)\right] d \theta_{1 k} d \theta_{k 1} .
\end{aligned}
$$

The above integral will vanish unless

$$
\left\{\begin{array}{l}
\alpha_{11}-\beta_{11}+\sum_{j, k \geq 2}\left(-\alpha_{j k}+\beta_{j k}\right)=0 \\
\sum_{j=1}^{n}\left(\alpha_{j k}-\beta_{j k}\right)=0, \sum_{j=1}^{n}\left(\alpha_{k j}-\beta_{k j}\right)=0, \quad(k=2, \ldots, n) .
\end{array}\right.
$$


This proves our first assertion. Multiplying the first eqution by 2 and adding all together, we get

$$
\sum_{j=1}^{n}\left(\alpha_{j 1}+\alpha_{1 j}\right)=\sum_{j=1}^{n}\left(\beta_{j 1}+\beta_{1 j}\right)
$$

For $k=2,3, \ldots, n$, we note that

$$
\begin{aligned}
& \sum_{j=1}^{n}\left(\alpha_{j k}+\alpha_{k j}\right)-\sum_{j=1}^{n}\left(\beta_{j k}+\beta_{k j}\right) \\
& =\sum_{j=1}^{n}\left(\alpha_{j k}-\beta_{j k}\right)+\sum_{j=1}^{n}\left(\alpha_{k j}-\beta_{k j}\right)=0
\end{aligned}
$$

and

$$
2|\alpha|=\sum_{k, j=1}^{n}\left(\alpha_{j k}+\alpha_{k j}\right)=\sum_{j, k=1}^{n}\left(\beta_{j k}+\beta_{k j}\right)=2|\beta| .
$$

Thus the proof is completed.

COROLLARY. Suppose $\alpha, \beta$ are two $n^{2}$-tuples of nonnegative integers satisfying the conditions in Proposition 5. Then

$$
\prod_{j, k=1}^{n}\left(\lambda_{j} \lambda_{k}\right)^{\alpha_{j k}}=\prod_{j, k=1}^{n}\left(\lambda_{j} \lambda_{k}\right)^{\beta_{j k}}
$$

for any numbers $\lambda_{1}, \lambda_{2}, \ldots, \lambda_{n}$.

PROOF OF THE THEOREM. Let

$$
N_{\{M\}}(t \Lambda)=C(k, n) \int_{D_{n}} \operatorname{det}\left(E-W^{t} \bar{W}\right)^{k-2 n} \operatorname{det}\left(E-t^{2} \bar{\Lambda} W \bar{\Lambda}^{t} \bar{W}\right)^{-k} d W
$$

with $0<t<1$. If we can prove

$$
N_{\{M\}}(t \Lambda)=\prod_{j, k=1}^{n}\left(1-t^{2} \bar{\lambda}_{j} \bar{\lambda}_{k}\right)^{-1}
$$

then we get

$$
\begin{aligned}
C(k, n) \int_{D_{n}} \operatorname{det}(E & \left.-W^{t} \bar{W}\right)^{k-2 n} \operatorname{det}\left(E-\bar{\Lambda} W \bar{\Lambda}^{t} \bar{W}\right)^{-k} d W \\
& =\prod_{j, k}^{n}\left(1-\bar{\lambda}_{j} \bar{\lambda}_{k}\right)^{-1}
\end{aligned}
$$

by letting $t$ approach 1 . Now, we shall prove (A).

Let $S$ be the index set of all $n^{2}$-tuples of integers $\alpha=\left[\alpha_{j k}\right], \alpha_{j k} \geq 0$. Consider all monomials $a_{\alpha}(W)=W^{\alpha}, \alpha \in S$, which are arranged in such order that their degrees are nondecreasing. By an argument similar to [7, p. 188], we can prove that $\left\{a_{\alpha}(W) \mid \alpha \in S\right\}$ is a complete system in $H\left(k, D_{n}\right)$ in the sense that if $f \in H\left(k, D_{n}\right)$ and

$$
\int_{D_{n}} \operatorname{det}\left(E-W^{t} \bar{W}\right)^{k-2 n} a_{\alpha}(W) \overline{f(W)} d W=0 \quad \forall \alpha \in S
$$


then $f(W)=0$. This system precisely consists of all terms in the power series expansion

$$
\prod_{j, k=1}^{n}\left(1-w_{j k}\right)^{-1}=\prod_{j, k=1}^{n}\left(1+w_{j k}+\cdots+w_{j k}^{m}+\cdots\right), \quad\left|w_{j k}\right|<1 .
$$

Of course, $\left\{a_{\alpha}(W)=W^{\alpha} \mid \alpha \in S\right\}$ is a linear independent set in $H\left(k, D_{n}\right)$. By the well-known Gram-Schmidt orthogonalization process, we can construct an orthonormal basis $\left\{\psi_{\alpha}(W) \mid \alpha \in S\right\}$ from $\left\{a_{\alpha}(W) \mid \alpha \in S\right\}$. Proposition 5 and its corollary then imply that the basis $\left\{\psi_{\alpha}(W) \mid \alpha \in S\right\}$ has the following properties:

(1) $\psi_{\alpha}(W)$ is a finite linear combination of monomials of degree $|\alpha|$.

(2) $\psi_{\alpha}\left(t^{2} \bar{\Lambda} W \bar{\Lambda}\right)=t^{2|\alpha|} \prod_{j, k=1}^{n}\left(\bar{\lambda}_{j} \bar{\lambda}_{k}\right)^{\alpha_{j k}} \cdot \psi_{\alpha}(W)$.

Choose $\left\{\psi_{\alpha}(W) \mid \alpha \in S\right\}$ as an orthonormal basis of $H\left(k, D_{n}\right)$ and note that

$$
C(k, n) \operatorname{det}\left(E-W^{t} \bar{W}_{1}\right)^{-k}=K\left(W, W_{1}\right)
$$

is a kernel function of $H\left(k, D_{n}\right)$. By Proposition 1 we then have

$$
\begin{aligned}
C(k, n) \operatorname{det}\left(E-t^{2} \bar{\Lambda} W \bar{\Lambda}^{t} \bar{W}\right)^{-k} & =\sum_{\alpha \in S} \psi_{\alpha}\left(t^{2} \bar{\Lambda} W \bar{\Lambda}\right) \overline{\psi_{\alpha}(W)} \\
& =\sum_{\alpha \in S} t^{2|\alpha|} \prod_{p, q=1}^{n}\left(\bar{\lambda}_{p} \bar{\lambda}_{q}\right)^{\alpha_{p q}} \psi_{\alpha}(W) \overline{\psi_{\alpha}(W)} .
\end{aligned}
$$

Multiply both sides with $\operatorname{det}\left(E-W^{t} \bar{W}\right)^{k-2 n}$ and integrate on $D_{n}$ to get

$$
\begin{aligned}
N_{\{M\}}(t \Lambda) & =\sum_{\alpha \in S} t^{2|\alpha|} \prod_{j, k=1}^{n}\left(\bar{\lambda}_{j} \bar{\lambda}_{k}\right)^{\alpha_{j k}} \\
& =\prod_{j, k=1}^{n}\left(1-t^{2} \bar{\lambda}_{j} \bar{\lambda}_{k}\right)^{-1}
\end{aligned}
$$

by the orthnormality of $\left\{\psi_{\alpha}(W) \mid \alpha \in S\right\}$. This proves our assertion in (A) and hence completes our proof.

REMARK 1. Note that for $0<t<1$, the integrand in $N_{\{M\}}(t \Lambda)$ is absolutely integrable and it can be integrated term by term after its decomposition as a Bergmann kernel function. However, it is not permissible for the integrand of $N_{\{M\}}(\Lambda)$ to do so.

REMARK 2. The Gram-Schmidt orthogonalization process is applied to monomials of the same degree since monomials of different degrees are orthogonal to each other by Proposition 5. Furthermore, we assume $\psi_{\alpha}(W)$ is the function obtained from $W^{\alpha}$ by this process.

5. Generalizations and applications. We shall generalize the evaluation of the integral is our Theorem to cases as follows:

(1) The integrand $\operatorname{det}\left(E-\bar{\Lambda} W \bar{\Lambda}^{t} \bar{W}\right)^{-k}$ is changed into a general form $\operatorname{det}\left(E-\bar{\Lambda}_{1} W \bar{\Lambda}_{2}^{t} \bar{W}\right)^{-k}$ with $\Lambda_{1}, \Lambda_{2}$ in $U(n)$, the unitary group.

(2) The domain $D_{n}$ is changed into the hyperbolic space of $p \times q$ matrices defined by

$$
D_{p, q}: W \in M_{p, q}(\mathbf{C}), \quad E_{q}-{ }^{t} \bar{W} W>0 .
$$


Here $M_{p, q}(\mathbf{C})$ is the set of all $p \times q$ matrices over $\mathbf{C}$ and $E_{q}$ is the unit matrix of $M_{q}(\mathbf{C})$.

For the first generalization, we then have the following

Proposition 6. Let $\Lambda_{1}=\operatorname{diag}\left[\lambda_{1}, \ldots, \lambda_{n}\right], \Lambda_{2}=\operatorname{diag}\left[\lambda_{n+1}, \ldots, \lambda_{2 n}\right]$, with $\lambda_{j}(j=1,2, \ldots, 2 n)$ roots of unity and $\lambda_{j} \lambda_{n+k} \neq 1$ for all $1 \leq j, k \leq n$, and let

$$
I=C(k, n) \int_{D_{n}} \operatorname{det}\left(E-W^{t} \bar{W}\right)^{k-2 n} \operatorname{det}\left(E-\bar{\Lambda}_{1} W \bar{\Lambda}_{2}^{t} \bar{W}\right)^{-k} d W \quad(k>4 n-2) .
$$

Then

$$
I=\prod_{j, k=1}^{n}\left(1-\bar{\lambda}_{j} \bar{\lambda}_{n+k}\right)^{-1}
$$

PROOF. The proof follows from a slight change in our proof of the Theorem. Conditions in Proposition 5 imply

$$
\sum_{j=1}^{n}\left(\alpha_{j k}-\beta_{j k}\right)=\sum_{j=1}^{n}\left(\alpha_{k j}-\beta_{k j}\right)=0 \quad(k=1,2, \ldots, n) .
$$

Let $\psi_{\alpha}(W), \alpha \in S$, be the function obtained from $W^{\alpha}$ with a Gram-Schmidt orthogonalization process. Then we have

$$
\begin{aligned}
\psi_{\alpha}\left(\bar{\Lambda}_{1} W \bar{\Lambda}_{2}\right) & =\prod_{j=1}^{n} \bar{\lambda}_{j}^{a(j)} \prod_{k=1}^{n} \bar{\lambda}_{n+k}^{b(k)} \cdot \psi_{\alpha}(W) \\
& =\prod_{j, k=1}^{n}\left(\bar{\lambda}_{j} \bar{\lambda}_{n+k}\right)^{\alpha_{j k}} \cdot \psi_{\alpha}(W)
\end{aligned}
$$

with $a(j)=\sum_{k=1}^{n} \alpha_{j k}$ and $b(k)=\sum_{j=1}^{n} \alpha_{j k}$. It follows

$$
\begin{aligned}
I & =\lim _{t \rightarrow 1} \sum_{\alpha \in S}\left(t^{2} \bar{\lambda}_{j} \bar{\lambda}_{n+k}\right)^{\alpha_{j k}} \\
& =\lim _{t \rightarrow 1} \prod_{j, k=1}^{n}\left(1-t^{2} \bar{\lambda}_{j} \bar{\lambda}_{n+k}\right)^{-1}=\prod_{j, k=1}^{n}\left(1-\bar{\lambda}_{j} \bar{\lambda}_{n+k}\right)^{-1} .
\end{aligned}
$$

Now we consider the second generalization. Let $\mathbf{F}$ be any imaginary quadratic field and define an algebraic group $G_{p, q}$ over $Q$ as follows:

$$
\left(G_{p, q}\right)_{Q}=\left\{\left.M \in S L_{p+q}(F)\right|^{t} \bar{M} R M=R, R=\left[\begin{array}{cc}
E_{p} & 0 \\
0 & -E_{q}
\end{array}\right]\right\}
$$

and $\left(G_{p, q}\right)_{\mathbf{R}}=S U(p, q)$. The group $S U(p, q)$ operates on the bounded domain $D_{p, q}$ by the action

$$
M: Z \rightarrow M(Z)=(A Z+B)(C Z+D)^{-1}, \quad M=\left[\begin{array}{cc}
A & B \\
C & D
\end{array}\right] \text { in } S U(p, q) .
$$

Here $M$ is so decomposed that $A, B, C$ and $D$ are $p \times p, p \times q, q \times p$ and $q \times q$ matrices respectively.

Let $\Gamma$ be a discrete subgroup of $G_{\mathbf{R}}$ such that $\Gamma \backslash G_{\mathbf{R}}$ has definite volume with respect to the invariant measure $\operatorname{det}\left(E_{q}-{ }^{t} \bar{W} W\right)^{-p-q} d W$. For positive integer 
$k$, we let $S(k ; \Gamma)$ be the vector space of the holomorphic function $f(W)$ on $D_{p, q}$ satisfying the conditions:

(1) $f(\gamma(W))=\operatorname{det}(C W+D)^{k} f(W)$ for all $\gamma=\left[\begin{array}{ll}A & B \\ C & D\end{array}\right] \in \Gamma$.

(2) $\left[\operatorname{det}\left(E_{q}-{ }^{t} \bar{W} W\right)\right]^{k / 2} f(W)$ is bounded in $D_{p, q}$.

A function $f$ in $S(k ; \Gamma)$ is called a cusp form of weight $k$. A standard argument [6] shows that if $k>2(p+q-1)$, then $S(k ; \Gamma)$ is a finite dimensional vector space. Furthermore, its dimension can be calculated via the Selberg trace formula. An element $M$ in $\Gamma$ is regular elliptic if $M$ is conjugate in $G_{\mathbf{R}}$ to $\Lambda_{p} \times \bar{\Lambda}_{q}=$ $\operatorname{diag}\left[\lambda_{1}, \ldots, \lambda_{p}, \bar{\lambda}_{p+1}, \ldots, \bar{\lambda}_{p+q}\right] \in S\left(U_{p} \times U_{q}\right)$ and $\lambda_{j} \lambda_{p+q} \neq 1$ for all $1 \leq j \leq$ $p, 1 \leq k \leq q$. This is equivalent to saying that $M$ has an isolated fixed point on $D_{p, q}$.

With these preparations, we now have the following

Proposition 7. Suppose $M \in \Gamma$ and is conjugate in $G_{\mathbf{R}}$ to $\Lambda_{p} \times \bar{\Lambda}_{q}=$ $\operatorname{diag}\left[\lambda_{1}, \ldots, \lambda_{p}, \bar{\lambda}_{p+1}, \ldots, \bar{\lambda}_{p+q}\right] \in S\left(U_{p} \times U_{q}\right)$ with $\lambda_{j} \lambda_{p+r} \neq 1$ for all $1 \leq j \leq$ $p, 1 \leq r \leq q$. Then the contribution of elements in $\Gamma$ which are conjugate in $\Gamma$ to $M$, to $\operatorname{dim}_{\mathrm{C}} S(k ; \Gamma)(k>2(p+q-1))$, is given by

$$
N_{\{M\}}=\left|C_{M, \mathbf{z}}\right|^{-1} \prod_{s=1}^{q} \bar{\lambda}_{p+s}^{k} \cdot \prod_{j=1}^{p} \prod_{r=1}^{q}\left(1-\bar{\lambda}_{j} \bar{\lambda}_{p+r}\right)^{-1}
$$

Here $\left|C_{M, \mathbf{Z}}\right|$ is the order of $C_{M, \mathbf{Z}}$ which is the centralizer of $M$ in $\bar{\Gamma}$, the quotient of $\Gamma$ by its center.

PROOF. Let $H\left(k ; D_{p, q}\right)$ be the vector space of holomorphic functions which are square integrable on $D_{p, q}$ with respect to the measure $\operatorname{det}\left(E_{q}-{ }^{t} \bar{W} W\right)^{k-p-q} d W$. From the argument of $[\mathbf{7}]$ or the explicit formula given in $[\mathbf{9}]$, we get that

$$
K\left(W_{1}, W_{2}\right)=C(k ; p, q) \operatorname{det}\left(E_{q}-{ }^{t} \bar{W}_{2} W_{1}\right)^{-k}
$$

with

$$
C(k ; p, q)=\pi^{-p q} \prod_{j=0}^{p-1} \prod_{r=0}^{q-1}(k-p-q+1+j+r)
$$

the kernel function of $H\left(k ; D_{p, q}\right)$. Also we note that the set of monomials in $W=$ $\left[w_{j r}\right](j=1, \ldots, p, r=1, \ldots, q)$ is an independent set as well as a complete system in $H\left(k ; D_{p, q}\right)$. Hence we can apply the Gram-Schmidt orthogonalization process to this set and get an orthonormal basis of $H\left(k ; D_{p, q}\right)$. The orthogonal relations in Proposition 5 still exist if we introduce the same coordinates for $D_{p, q}$ as we have done for $D_{n}$ in Proposition 4. Consequently, we prove that

$$
\begin{aligned}
I_{p, q} & =C(k ; p, q) \int_{D_{p, q}} \operatorname{det}\left(E_{q}-{ }^{t} \bar{W} W\right)^{k-p-q} \operatorname{det}\left(E_{q}-\bar{\Lambda}_{q}{ }^{t} \bar{W} \bar{\Lambda}_{p} W\right)^{-k} d W \\
& =\prod_{j=1}^{p} \prod_{r=1}^{q}\left(1-\bar{\lambda}_{j} \bar{\lambda}_{p+r}\right)^{-1} .
\end{aligned}
$$


On the other hand, a standard argument (to change the order of integration and summation) [13] shows that the contribution $N_{\{M\}}$ is given by

$$
\begin{aligned}
N_{\{M\}} & =\left|C_{M, \mathbf{z}}\right|^{-1}\left(\operatorname{det} \Lambda_{q}\right)^{-k} I_{p, q} \\
& =\left|C_{M, \mathbf{z}}\right|^{-1} \prod_{s=1}^{q} \bar{\lambda}_{p+s}^{k} \cdot \prod_{j=1}^{p} \prod_{r=1}^{q}\left(1-\bar{\lambda}_{j} \bar{\lambda}_{p+r}\right)^{-1} .
\end{aligned}
$$

This proves our assertion.

REMARK. Proposition 6 can be applied to cases which may be left out by our main Theorem.

\section{REFERENCES}

1. Hel Braun, Hermitian modular functions, Ann. of Math. (2) 50 (1949), 827-855.

2. Hermitian modular functions. III. The Hermitian modular group, Ann. of Math. (2) 53 (1951), 143-180.

3. Minking Eie, Dimension formulas for the vector spaces of Siegel's modular cusp forms of degree two and degree three, Thesis, University of Chicago, 1982, pp. 1-246.

4. _ Dimensions of spaces of Siegel cusp forms of degree two and three, Mem. Amer. Math. Soc. No. 304 (1984), pp. 1-185.

5. __ Contributions from conjugacy classes of regular elliptic elements in $\operatorname{Sp}(n, \mathbf{Z})$ to the dimension formula, Trans. Amer. Math. Soc. 285 (1984), 403-410.

6. R. Godement, Généralités sur les formes modulaires. I, II, Séminaire Henri Cartan, 10e années, 1957, 1958.

7. L. K. Hua, On the theory of functions of several complex variables. I, II, III, Amer. Math. Soc. Transl. 32 (1962), 163-263.

8. __ Inequalities involving determinants, Amer. Math. Soc. Transl. 32 (1962), 265-272.

9. Suehiro Kato, $A$ dimension formula for a certain space of automorphic forms of $S U(p, q)$, Math. Ann. 266 (1984), 457-477.

10. Hans Maass, Siegel's modular forms and Dirichlet series, Lecture Notes in Math., vol. 216, Springer-Verlag, Berlin and New York, 1971.

11. S. Helgason, Differential geometry and symmetric spaces, Academic Press, New York, 1962.

12. George W. Machkey, Unitary group representation in physics, probability and number theory, Benjamin, New York, 1978.

13. A. Selberg, Harmonic analysis and discontinuous groups in weakly symmetric Riemannian spaces with applications to Dirichlet series, J. Indian Math. Soc. 20 (1956), 47-87.

14. Hideo Shimizu, On discontinuous groups operating on the product of the upper half plane, Math. Ann. 177 (1963), 33-71.

15. C. L. Siegel, Lectures on quadratic forms, Tata Institute of Fundamental Research, Bombay, 1967.

Institute of mathematics, ACademia Sinica, Nankang, Taipei, Taiwan, RePUBLIC OF CHINA

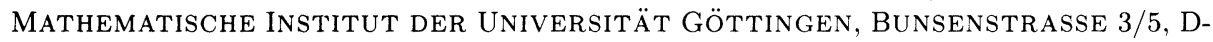
3400 Göttingen, Federal Republic of Germany 